\title{
The Effect of Upper Limb Elevation on Limb Edema and Central Venous Pressure in ICU Patients
}

\author{
Mehdi Ahmadinejad, ${ }^{1}$ Faezeh Heravi, ${ }^{2}$ Mansooreh Aziz Zadeh, ${ }^{3, *}$ and Yoones Jahani ${ }^{4}$ \\ ${ }^{1}$ Department of Anesthesiology, Kerman University of Medical Sciences, Kerman, IR Iran \\ ${ }_{2}^{2}$ Department of Nursing, Razi School of Nursing and Midwifery, Kerman University of Medical Sciences, Kerman, IR Iran \\ 3 Neuroscience Research Center, Faculty of Internal Diseases and Surgery, Kerman University of Medical Sciences, Kerman, IR Iran \\ ${ }^{4}$ Department of Statistics and Epidemiology, Kerman University of Medical Sciences, Kerman, IR Iran \\ ${ }^{*}$ Corresponding Author: Mansooreh Aziz Zadeh, Neuroscience Research Center, Faculty of Internal Diseases and Surgery, Kerman University of Medical Sciences, Kerman, IR Iran. \\ E-mail:m_forozy@kmu.ac.ir
}

Received:September 10, 2014; Revised: October 11, 2014; Accepted: October 20, 2014

\begin{abstract}
Background: Patients admitted to ICU are susceptible to different complications, such as extremities edema. Proper nursing intervention is one of the important aspects of ICU patient's care.

Objectives: The aim of this study was to determine the effects of hand elevation to reduce hand edema and intravenous volume of ICU patients.

Patients and Methods: This experimental clinical study was conducted on 40 ICU patients in hospitals affiliated to Kerman university of medical sciences, Kerman, Iran. During five consecutive days, we randomly elevated one hand of patients with edema, using a 30-degree inclined plane and fixed it for 30 minutes. In each session, before and after limb elevation, central venous pressure (CPV), size of the wrist and the midsection of the arm were measured and recorded.

Results: Elevating upper limb was effective to reduce hand edema and increasing CVP of ICU patients $(\mathrm{P}<0.05)$. In addition, there was a meaningful association between gender and reduction of arm edema $(P=0.04)$, and between GCS and wrist edema $(P=0.02)$.

Conclusions: Raising upper limb as a low expense method without any complications could be efficient to reduce hand edema of ICU patients and improve effective intravascular volume.

Keywords: Edema; Limb; Pressure
\end{abstract}

\section{Background}

Edema is an abnormal accumulation of fluid in the interstitium, which are locations beneath the skin or in one or more body cavities $(1,2)$. Swelling is seen clearly when about $2.5-3 \mathrm{~L}$ is accumulated in the interstitium space (3) locally or generally (4). Usually, accumulated fluid is removed by the lymphatic system and discharged into the veins, but in cases which edema is too much for the lymphatic drainage system, the fluid remains in the interstitium space (5). Generally, there are four main reasons for edema: decreased plasma oncotic pressure, increased plasma hydrostatic pressure, increased vascular permeability and decreased lymphatic drainage $(4,6)$. Decreased plasma oncotic pressure in ICU patients could result from undernourishment after surgery, burning, head injury, liver diseases $(3,4)$ or excessive proteinuria $(3,6)$. Capillary hydrostatic pressure increases with raising of intravenous pressure, in some situations such as: volume overload and veins obstruction (3). Increased permeability of the vessels in generalized inflammations could cause local or general edema. In fact, permeability of capillary system increases in in- jured areas, then proteins easily enter the interstitium space creating an osmotic pressure absorbing fluid. In addition, permeability of capillary system increases in ischemic and infectious disorders due to the release of inflammatory cytokines. According to what described before about the different causes of edema, the disorder could affect different parts of body, as Giodis mentioned that hand edema, is a recurrent disorder after trauma or surgery followed by complications such as pain (7), change in range of motion and dryness of the hand joints, limitation of the kinetic domain and atrophy (8). Moreover, edema impairs the movement of nutrients and waste products between blood capillaries and cells $(1,9)$, which could expose the patient to secondary complications such as infection, delay in wound healing, cellular damages (6) and pressure ulcers due to reduction of lymphatic drainage (10). Medical and nonmedical modalities are used to treat edema. Medical treatments as diuretics, improve symptoms of edema, but could induce renal failure and decrease the blood supply to the tissues, due to reduction of intravascular volume

Copyright (C) 2015, Shahid Beheshti University of Medical Siences. This is an open-access article distributed under the terms of the Creative Commons AttributionNonCommercial 4.0 International License (http://creativecommons.org/licenses/by-nc/4.0/) which permits copy and redistribute the material just in noncommercial usages, provided the original work is properly cited. 
(3). Nonmedical treatments include active and passive exercises, cryotherapy and diathermy, low-voltage electric stimulation, massage and different methods creating external pressure (1). One of the suggested methods for reducing patients' hand edema after surgery, trauma and hand paralysis is elevating the upper limb (6). In this method the hand is elevated higher than elbow, and elbow higher than shoulder (8). The limb is elevated higher than the heart level and this could reduce the intravenous pressure by the gravity; following which the filtration pressure of the capillaries is also reduced (1). Kawasaki et al. conducted a study with the goal of evaluating the different positions on lower limb blood circulation and edema. They concluded that in sitting position and appropriate leg position, skin perfusion pressure was increased significantly compared with other positions in both healthy adults and critical patients. These results suggest that sitting position is effective to keep an appropriate blood stream of lower limbs not only in healthy adults but also in patients with critical limb ischemia (11). Therefore, it seems that limb elevation could be effective to prevent and reduce limb edema. A study was conducted to compare the effects of limb elevation with and without using the pneumatic massage system on reducing foot edema, in patients with knee sprain. This study showed that both methods reduced the patients' edema, but their combination was more effective (12). Whereas in another study, elevating the hand and arm had no significant effect on reducing patients' hand edema (13).

\section{Objectives}

Considering the prevalence of limb edema in ICU and its complications that could impose high expenses on patients' family and healthcare system, and inconsistency of existing results, we conducted this research to evaluate the effect of upper limb elevation on limb edema and central venous pressure in patients admitted to ICU.

\section{Patients and Methods}

This was a one-way blind clinical experimental study conducted on ICU patients of Shahid Bahonar hospital (affiliated to Kerman university of medical sciences). The study was approved in the ethical committee of the research administration of Kerman medical university (code of K/92/179). This research was registered in the international center of Iranian registry of clinical trials (with the code of 201307155426N6). The Sample volume was determined 40 subjects considering the first type error of $5 \%(\%=\alpha 5)$ and the test power of $\% 80(1-\beta-\% 80)$ according to the study of Bagheri et al. Data gathering tool was a questionnaire consisting of three parts as demographic information, information about the disease and the template for recording the degree of edema. We randomly chose the hand to be kept elevated. The inclusion criterion was all patients with severe head injury (GCS <
8) and upper limb pitting edema (according to the scale of the Guelph General Hospital Congestive Heart Failure Pathway). The exclusion criteria were deep venous thrombosis of the upper limb, soft tissue injury, broken bone or infection in the understudy limb, placement of central venous catheter in the subclavian vein of the same side, mastectomy, chronic heart failure, liver and renal disease, hypothyroidism and increased level of consciousness (GCS > 8) before the completion of the study (5 days). After selection of patients, upper limb edema was assessed and recorded according to the scale of pitting edema. The size of the wrist and the midsection of the patients' arm were measured by a tapeline, and in the next step, the patient's hand was fixed on a 30-degree inclined plane for 30 minutes with the patient in supine position. We used tapeline measure and inclined plane after acquiring standard certification from the Sepehr Laboratory in Tehran (standard code 92S3144 for the tape measure and the standard code $92 \mathrm{~S} 03143$ for the inclined plane). On the other hand, before any intervention, the patient's central venous pressure (CPV) was measured and recorded by a skillful ICU nurse who was blind to hand elevation. This was performed for every patient during five consecutive days and in each session before and after doing the intervention, the CVP, wrist and the midsection of the arm size and edema were measured and recorded. Maintenance fluid was only perfused during the study, so that it could not affect CVP. During each session, capillary filling and skin integrity were evaluated by the researcher. For five sessions, the routine care and daily physiotherapy of the upper limbs were performed for all patients. To meet the ethical principles, written subscriptions were obtained. It was explained that there was no obligation for participating in the study. After gathering data, the information was analyzed using SPSS ver. 21(IBM Company, USA) and the central indices and distribution of demographic variables were determined. To evaluate the association between limb elevation and CVP change, the wrist and arm edema, the Wilkakson test was used (we calculated the average amounts of CVP and wrist and arm edema before and after five consecutive days intervention and then their average values were compared). To evaluate the association between reduction of the upper limb edema and other variables (such as gender, GCS, etc.), Man Whitney, Kruskal Wallace tests and Spearman coefficient were used.

\section{Results}

Of 40 patients, $32(80 \%)$ were male, and $8(20 \%)$ were female with the mean age of $46.2 \pm 21.3$. In total, $27.5 \%$ had first-degree edema, 52.5\% second degree, and 20\% third degree. regarding the level of consciousness, the least GCS was equal to 3 and the most was 8 (with the mean of $5 \pm 1.8$ ). The minimum acute physiological status and chronic health score (APACHEII) was 40 and the maximum was 91 (with the mean of $62.1 \pm 10.22$ ). The minimum time of hospitalization was three days before en- 
tering the study and the maximum was 100 days (17.5 \pm 20.9). In addition, the minimum level of serum albumin was $2.1 \mathrm{mg} / \mathrm{dL}$ and the maximum was $4.8 \mathrm{mg} / \mathrm{dL}$ (with the mean of $3.92 \pm 0.52$ ). Data analysis indicated that in all of the sessions after elevating the upper limb, the patients' CVP was significantly increased and the average wrist and arm edema were decreased $(\mathrm{P}<0.05)$, which is presented in Table 1. According to this study, there was no significant association between decrease in wrist and arm edema and demographic variables such as age, duration of hospitalization, level of serum albumin and the APACHE
II (acute physiology, age and chronic health evaluation score) score. Whereas, there was a significant association between gender and decreased arm edema; the amount of decrease in women was more than men $(P=0.04)$, but there was no meaningful difference between men and women regarding wrist edema $(\mathrm{P}=0.52)$. A significant association was observed between decrease in wrist edema and GCS $(\mathrm{P}=0.02)$ (patients with more GCS showed more decreasing of wrist edema), but this association was not meaningful for arm edema $(\mathrm{P}=0.76)$. The results were reported in the Tables 2 and 3.

Table 1. Comparison Between Decrease in Wrist and Arm Edema, Central Venous Pressure, Before and After the Intervention After Five Sessions

\begin{tabular}{lccc}
\hline Variable & Mean \pm SD & Median (Safe Distance) & P Value \\
\hline Decrease in arm edema & $0.59 \pm 0.62$ & $0.62(0.44-0.71)$ & $<0.0001$ \\
Decrease in wrist edema & $0.49 \pm 0.29$ & $0.43(0.35-0.51)$ & $<0.0001$ \\
$\begin{array}{l}\text { Decrease in the central } \\
\text { venous pressure }\end{array}$ & $1.87 \pm 1.35$ & $1.56(2.4-1.13)$ & $<0.0001$ \\
\hline
\end{tabular}

Table 2. The Association Between Decrease in Edema According to Gender and Diagnosis

\begin{tabular}{|lccc}
\hline Variable & Mean \pm SD & Median (Safe Distance 95\%) & PValue \\
\hline $\begin{array}{l}\text { The Average Decrease in Arm Edema } \\
\text { Gender }\end{array}$ & & & \\
\hline Male & $0.52 \pm 0.66$ & $0.54(0.83-0.25)$ & 0.04 \\
\hline Female & $0.87 \pm 0.29$ & $0.82(1.08-0.56)$ & 0.82 \\
\hline Diagnosis & & $0.64(0.94-0.34)$ & $0.66(1.19-0.12)$ \\
\hline Only neurosurgery & $0.63 \pm 0.65$ & $0.57(1.21-0.08)$ & \\
\hline Neurosurgery and internal surgery & $0.59 \pm 0.48$ & & 0.52 \\
\hline Neurosurgery and internal surgery & $0.37 \pm 0.61$ & $0.41(0.55-0.27)$ \\
\hline $\begin{array}{l}\text { The Average Decrease in Wrist Edema } \\
\text { Gender }\end{array}$ & & $0.45(0.62-0.28)$ & \\
\hline Male & $0.49 \pm 0.31$ & $0.44(0.56-0.32)$ \\
\hline Female & $0.5 \pm 0.19$ & $0.32(0.53-0.11)$ \\
\hline Diagnosis & & $0.51(1.01-0.02)$ \\
\hline Only neurosurgery & $0.5 \pm 0.25$ & \\
\hline Neurosurgery and internal surgery & $0.35 \pm 0.19$ & 0.34 \\
\hline Neurosurgery and internal surgery & $0.59 \pm 0.48$ & & \\
\hline
\end{tabular}

Table 3. The Association Between Decrease in Edema and Demographic Variables and Disease Characteristics a

\begin{tabular}{lcccc}
\hline Variable & \multicolumn{2}{c}{ The Decrease in Wrist Edema } & \multicolumn{2}{c}{ The Decrease in Arm Edema } \\
\cline { 2 - 5 } & Coefficient Factor & P Value & Coefficient Factor & P Value \\
\hline Age & 0.05 & 0.74 & 0.16 & 0.31 \\
The days hospitalized & 0.04 & 0.82 & -0.16 & 0.34 \\
Albumin level & 0.09 & 0.58 & 0.13 & 0.41 \\
APACHE II Score & 0.12 & 0.45 & 0.04 & 0.79 \\
GCS & 0.36 & 0.02 & -0.05 & 0.76 \\
\hline
\end{tabular}

\footnotetext{
a Abbreviations: APACHE, Acute Physiology and Chronic Health Evaluation; GCS, Glasgow coma scale.
} 


\section{Discussion}

Upper limb elevation for 30 degrees increased CVP and reduced edema significantly. Extremity edema is a common problem in ICU patients leading to many complications such as pressure ulcer, reduced range of motion of joints, and some others.

Kawasaki et al. found that the circulation of lower limbs is better in hanged positions (in a lower level than heart), but staying for a long time in this position causes venous congestion, edema and delayed wound healing (11). Our study indicated that 30-degree elevation of upper limb could reduce the interstitium fluid and edema. As our study, Tsang et al. in a survey concluded that both lower limb elevation and using pneumatic compression system, can reduce foot edema but the elevating method was more effective than pneumatic compression system (12). There are some studies contrary to our results. For example, Fagan et al. conducted a study to evaluate the effects of upper limb elevation on patients' hand edema after carpal tunnel syndrome surgery and showed that limb elevation has no effects on reducing the hand edema (13). The difference between our results and Fagan et al. may be due different causes of edema. Patients in Fagan et al. study had limb soft tissue damages due to surgery but in our study the most common cause of edema was accumulation of excessive fluid in interstitium. In the recent years, some studies were conducted to evaluate the effect of gravity on limb edema. Boland RA and et al. conducted a study to compare three ways of hand elevation on reduction of hand edema; 30-degree angle, horizontal elevation, and 30-degree head of the bed elevation. they concluded that 30-degree elevation of upper limb is more effective than the other two methods (14), which is consistent with our results, but they used volumetric device to evaluate the edema and we could not use it because our patients were in coma state. Furthermore, Baker et al. conducted a research to evaluate the effect of limb effect on edema after fasciotomy operation. They concluded that although hand edema was reduced in the group receiving this intervention, but there was no significant difference between the two groups (15). However in this research, they elevated the hand only once, but we elevated the hand in five consecutive days. Maybe frequency of elevation could have a significant effect. We could not find a study evaluating the effects of elevating edematous limbs simultaneously on edema and CVP. Maintaining effective intravenous volume with the least amount of edema (to prevent complications such as compartment syndrome and intensification of ARDS) is of great importance in ICU patients. According to our results, it could be possible to prevent frequent infusion of intravenous fluid to maintain the effective circulating volume, by limbs elevation and returning the excessive interstitium fluid volume to the blood circulation. Based on the results of the present study, there was a meaningful association between gender and decrease of edema; decrease of edema was more in women than men. Moreover, our study indicated a significant association between decrease of wrist edema and GCS; higher GCS was related to more reducing wrist edema. the point is that the kinetic responses, are one of the main factors for evaluating the GCS and with increasing the amount of activity, they gain higher scores in this mode, so this relationship association seems to be logical; but no similar study was found to compare the association between decrease of limb edema and patients' GCS and gender. Accordingly, more extensive studies are needed to verify our findings. Elevating the upper limb is an effective method to reduce patients' hand edema increasing the effective intravascular volume. Most patients admitted to ICU have edema due to low level of consciousness and lack of movement. Therefore, we recommend limbs elevation in ICU patients with edema, because it is an effective, easy and inexpensive method.

\section{Acknowledgements}

The authors greatly appreciate the research administration of Kerman medical school, the staff and all of the ICU patients of Shahid Bahonar hospital in Kerman who honestly helped us in this research.

\section{Authors' Contributions}

Study concept and design: Ahmadi, Forouzi, Heravi. Acquisition of data: Ahmadi, Heravi Analysis and interpretation of data: Forouzi, Ahmadi, Harandi. Drafting of the manuscript: Ahmadi. Critical revision of the manuscript for important intellectual content: Ahmadi, Forouzi. Statistical analysis: Jahani. Administrative, technical, and material support: Heravi, Ahmadi, Forouzi, Harandi. Study supervision: Ahmadi, Forouzi.

\section{Funding/Support}

The funding organization is research vice chancellor of Kerman University of Medical Sciences.

\section{References}

1. Villeco JP. Edema: a silent but important factor. $J$ Hand Ther 2012;25(2):153-61.

2. Urden LD, Stacy KM, Lough ME. Thelan's critical care nursing diagnosis and management. St Louis: Mosby Elsevier; 2010.

3. Burton D, Rose BD. Pathophysiology and etiology of edema-II. 2000 Available from: http://www.pedneph.info.

4. Lotfi MT. [Lymphedema]. QJ Breast Disease. 2008;1:50-3.

5. Black JM, Hawks J. Text Book of Medical Surgical Nursing. New York: Elsevier; 2009.

6. Vasudevan SV, Melvin JL. Upper extremity edema control: rationale of the techniques. Am J Occup Ther.1979;33(8):520-3.

7. Mader K, Kirchner S, Wolfgarten B, Pennig D. Efficacy of the AV Impulse System versus cryotherapy in the reduction of postoperative oedema of the hand: a prospective randomised trial. Strategies Trauma Limb Reconstr. 2006;1(1):36-41.

8. Giudice ML. Effects of continuous passive motion and elevation on hand edema. Am J Occup Ther.1990;44(10):914-21. 


\section{Ahmadinejad M et al.}

9. Nemeth AJ, Falanga V, Alstadt SP, Eaglstein WH. Ulcerated edematous limbs: effect of edema removal on transcutaneous oxygen measurements. J Am Acad Dermatol.1989;20(2 Pt 1):191-7.

10. Krouskop TA, Reddy NP, Spencer WA, Secor JW. Mechanisms of decubitus ulcer formation--an hypothesis. Med Hypotheses. 1978;4(1):37-9.

11. Kawasaki T, Uemura T, Matsuo K, Masumoto K, Harada Y, Chuman $\mathrm{T}$, et al. The effect of different positions on lower limbs skin perfusion pressure. Indian J Plast Surg. 2013;46(3):508-12.

12. Tsang KK, Hertel J, Denegar CR. Volume Decreases After Elevation and Intermittent Compression of Postacute Ankle Sprains
Are Negated by Gravity-Dependent Positioning. J Athl Train. 2003;38(4):320-4.

13. Fagan DJ, Evans A, Ghandour A, Prabhkaran P, Clay NR. A controlled clinical trial of postoperative hand elevation at home following day-case surgery. J Hand Surg Br. 2004;29(5):458-60.

14. Boland RA, Adams RD. The effects of arm elevation and overnight head-up tilt on forearm and hand volume. $J$ Hand Ther. 1998;11(3):180-90.

15. Baker RP, Field J, Gozzard C, Wyatt MC, Robertson Y. Does postoperative hand elevation reduce swelling? A randomized study. $J$ Hand Surg Eur Vol. 2010;35(3):192-4. 